\title{
Ubicación, Dimensionamiento y Precio de Contrato Óptimo de Generación Distribuida en Sistemas de Distribución
}

\author{
Jesús M. López-Lezama, Luisa F. Buitrago y Fernando Villada \\ Grupo de Investigación GIMEL, Departamento de Ingeniería Eléctrica, Facultad de Ingeniería, Universidad \\ de Antioquia, Calle 70 No. 52-21, Medellín - Colombia (e-mail: jmaria.lopez@udea.edu.co; \\ luisa_fernanda1806@hotmail.com;fernando.villada@udea.edu.co).
}

Recibido Jun. 12, 2015; Aceptado Ago. 20, 2015; Versión final Ago. 28, 2015, Publicado Dic. 2015

\begin{abstract}
Resumen
En este trabajo se presenta una metodología para determinar la ubicación, precio de contrato y tamaño óptimo de generación distribuida (GD) en sistemas de distribución. En la metodología propuesta se supone la interacción de dos agentes: el propietario de la GD y el operador de la red de distribución. Por un lado, el propietario de la GD pretende maximizar sus ganancias al vender energía al operador de la red de distribución. Por otro lado, el operador de la red de distribución pretende minimizar los pagos incurridos en atender la demanda de la red. La interacción entre ambos agentes da lugar a un problema de programación binivel. El modelo equivalente para determinar el precio de contrato, ubicación y dimensionamiento óptimo de las unidades de GD que cumpla con las expectativas de ambos agentes es un problema de programación no lineal entero mixto que se resuelve mediante un algoritmo genético especializado. La metodología es puesta a prueba en un sistema de distribución de 85 barras mostrando resultados satisfactorios.
\end{abstract}

Palabras clave: generación distribuida; algoritmos genéticos; optimización; programación binivel.

\section{Location, Sizing and Optimal Contracting Price of Distributed Generation in Distribution Networks}

\begin{abstract}
This work presents a methodology for the optimal location, sizing and contract pricing of distributed generation (DG) in distribution systems. The proposed methodology is based on the interaction of two agents: the owner of the $D G$ and the distribution system operator. On one hand the owner of the DG aims at maximizing his profits from energy sales. On the other hand, the distribution system operator aims at minimizing the payments incurred in attending the network demand. The interaction between these two agents gives rise to a bi-level programming problem. The equivalent model to account for optimal location, pricing and sizing of DG, that accomplishes the expectative of both agents, corresponds to a mixed integer nonlinear programming problem which is solved by means of a specialized genetic algorithm. The methodology is tested on an 85-bus distribution system, showing satisfactory results.
\end{abstract}

Keywords: distributed generation; genetic algorithms; optimization; bi-level programming 


\section{INTRODUCCIÓN}

La generación de electricidad a pequeña escala, conocida como Generación Distribuida (GD), es cada vez más común en las redes de distribución. Esta tendencia ha sido motivada por diversos factores en los que se destaca la evolución de las tecnologías de generación a pequeña escala y la preferencia por el uso de recursos renovables para la generación de electricidad (Aghaei et al., 2012). Los beneficios de la GD han sido ampliamente reportados en la literatura técnica e incluyen el mejoramiento en el perfil de tensiones, la reducción de pérdidas, el aplazamiento de inversiones y el aumento en la confiabilidad del sistema (Soroudi et al., 2011). Sin embargo, vale la pena destacar que estos beneficios dependen de una correcta ubicación y dimensionamiento de las fuentes de GD. En este contexto, diferentes autores han propuesto metodologías para ubicar y/o dimensionar de forma apropiada la GD que será integrada en las redes de distribución. Las metodologías propuestas incluyen métodos analíticos (Hung et al., 2010), programación matemática (Kumar y Gao, 2010), algoritmos genéticos (Yuanyuan et al., 2014), optimización basada en colonias de partículas (Abu-Mouti y El-Hawary, 2011).

Un factor común en los estudios de ubicación y dimensionamiento óptimo de GD es que solo se tiene en cuenta el punto de vista de un único agente; bien sea el operador de la red de distribución o el inversionista en GD. Si bien muchos autores han abordado el problema desde el punto de vista multiobjetivo como (Singh y Verma, 2009) y (Shaaban et al., 2013), buscando encontrar una solución que cumpla con varios atributos a la vez; muy pocos han abordado el problema desde el punto de vista muti-agente. Ejemplos de este último abordaje se presentan en (Lopez-Lezama et al., 2011) y (Mobarakeh et al., 2013) donde se utiliza programación binivel para determinar únicamente el precio de contrato óptimo de GD. En ambos casos el problema del nivel inferior es reemplazado por sus condiciones de optimalidad, dando como resultado un problema equivalente de un solo nivel que puede ser resuelto mediante técnicas de programación matemática. Una revisión de modelos y métodos de solución para ubicar y dimensionar GD puede ser consultada en (Georgilakis y Hatziargyriou, 2013).

La principal diferencia del modelo propuesto en este artículo con otros publicados en la literatura técnica consiste en considerar no solo el precio de contrato sino también la ubicación y dimensionamiento de la GD en un esquema de programación binivel. En este caso se considera la interacción de dos agentes con diferentes intereses o funciones objetivo. Por un lado el propietario de la GD busca maximizar sus ganancias, mediante la venta de energía al operador de red, mientras que este último busca atender la demanda a mínimo costo. Para atender la demanda, el operador de la red de distribución puede comprar energía del mercado mayorista y/o de la GD. El problema del operador de la red consiste en determinar cuanta energía comprar de la GD y cuanta del mercado mayorista para minimizar el costo de atender la demanda. En este caso, el propietario de la GD debe anticiparse a la reacción del operador de red para maximizar sus ganancias. En la Fig. 1 se ilustra el problema binivel propuesto. El problema binivel presentado anteriormente es una generalización del Juego líder-seguidor de Stackelber. Por otro lado, desde el punto de vista matemático, un problema binivel corresponde a un problema de optimización matemática con restricciones de equilibrio (Mathematical Problema with Equilibrium Constraints - MPEC).

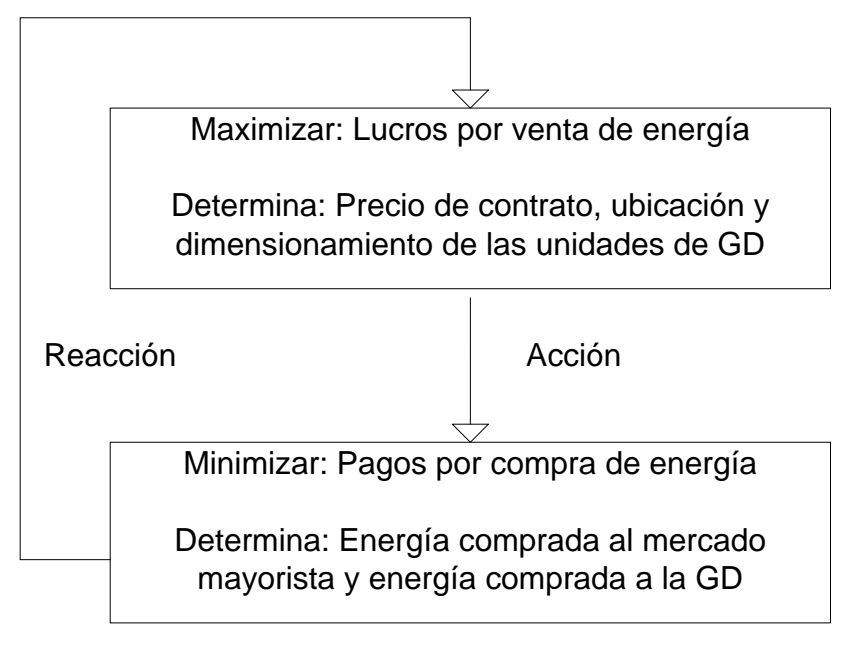

Propietario

de la GD

\section{Empresa}

Distribuidora

Fig. 1: Problema binivel propuesto.

Los esquemas binivel permiten modelar una relación jerárquica entre dos agentes y han sido aplicados en diferentes campos como los mercados eléctricos (Carrión et al., 2009), el problema de planeamiento de la expansión de la red (Garcés et al., 2009) y la confiabilidad en sistemas de potencia (Agudelo et al., 2014). Sin embargo, inclusive en su versión más simple (problemas del nivel superior e inferior lineales) el 
equivalente matemático de un solo nivel resulta ser un problema no lineal y no convexo, lo que justifica el uso de técnicas metaheurísticas para su solución, como la presentada en este artículo.

\section{MODELO MATEMATICO}

A continuación se describen los modelos matemáticos de los problemas de optimización del propietario de la GD y del operador de la red de distribución.

\section{Problema del nivel superior}

En el nivel superior del problema binivel se encuentra el propietario de la GD. Dado un número fijo de unidades de GD para instalar en la red, el propietario de la GD debe determinar el tamaño, ubicación y precio de venta que maximicen sus ganancias. El propietario de la GD debe tener en cuenta que el operador de red considera no solo el precio de venta sino el impacto de la GD como criterio para comprar energía. Dentro de su proceso de decisión, el propietario de la GD debe calcular y anticiparse a la reacción del operador de red. Las ecuaciones (1) a (3) describen este problema.

$\operatorname{Max} \sum_{t \in T} \sum_{j \in J} \Delta t\left\{\left(P C_{G D j}-C O_{G D j}\right) P_{G D j}(t)-C I_{G D j}(t) . P_{D G j}^{M a x}\right\}$

Sujeto a:

$P_{G D j}^{M i n} \leq P_{G D j}(t) \leq P_{G D j}^{M a x} \quad \forall j \in J ; \forall t \in T$

Reacción del operador de red

este caso $\mathrm{T}$ y $\mathrm{J}$ son conjuntos de intervalos de tiempo y cantidad de unidades de GD, respectivamente. $\Delta t$ corresponde a la longitud del intervalo de tiempo $\mathrm{t} ; \mathrm{PC}_{\mathrm{GDj}}$ es el precio de contrato de venta de energía de la unidad de $\mathrm{GD}$ j; $\mathrm{CO}_{\mathrm{GDj}}$ es el costo de operación de la unidad de $\mathrm{GD}$ j; $\mathrm{P}_{\mathrm{GDj}}(\mathrm{t})$ es el despacho de generación de la unidad de $G D \mathrm{j}$, en el intervalo $\mathrm{t} ; \mathrm{CI}_{\mathrm{GDj}}(\mathrm{t})$ es el costo de inversión de la unidad de $\mathrm{GD}$ j en el intervalo de tiempo $\mathrm{t}$; finalmente $\mathrm{P}_{\mathrm{DGj}}^{\mathrm{Max}}$ y $\mathrm{P}_{\mathrm{GDj}}^{\mathrm{Min}}$ son los límites mínimos y máximos de la unidad de $\mathrm{GD} \mathrm{j}$, respectivamente. Se debe resaltar que el despacho de generación es una decisión del operador de red, mientras el tamaño, ubicación y precio de contrato son decisiones del propietario de la GD.

\section{Problema del nivel inferior}

En el nivel inferior se encuentra el operador de red, que desea atender la demanda a mínimo costo. Con respecto al suministro de energía mediante unidades de GD, el operador de red debe tener en cuenta no solo el precio de la energía de estas unidades sino también el impacto que estas tienen en su red. Es decir, el único parámetro de decisión para el operador de red no es solo el precio del suministro sino el impacto de la GD en pérdidas, perfiles de tensión y congestión del sistema. Para tener en cuenta estas variables, el operador de la red de distribución debe realizar un despacho de carga óptimo. Las ecuaciones (4) a (11) corresponden a la formulación matemática del problema del nivel inferior. Note que este conjunto de ecuaciones modelan un despacho óptimo (reacción del operador de red) y corresponden a la restricción (3) del problema del nivel superior. Adicionalmente, la ubicación, dimensionamiento y precio de contrato de las unidades de GD son parámetros para este problema, pues han sido previamente definidas por el propietario de la GD.

$\operatorname{Min} \sum_{t \in T} \sum_{k \in K} \Delta_{t} \rho_{\mathrm{SE}_{\mathrm{k}}}(t) P_{S E k}(t)+\sum_{t \in T} \sum_{j \in J} \Delta_{t} P C_{G D_{j}}(t) P_{G D j}(t)$

$P_{G_{n}}(t)-P_{D_{n}}(t)-P_{n}(t)=0 ; \forall_{n} \in N, \forall_{t} \in T$

$Q_{G_{n}}(t)-Q_{D_{n}}(t)-Q_{n}(t)=0 ; \forall_{n} \in N, \forall_{t} \in T$

$P_{S E_{k}}^{M i n} \leq P_{S E_{k}}(t) \leq P_{S E_{k}}^{M a x} ; \forall_{k} \in K, \forall_{t} \in T$ 


$$
\begin{aligned}
& P_{G D_{j}}^{M i n}(t) \leq P_{G D_{j}}(t) \leq P_{G D_{j}}^{M a x} ; \forall_{j} \in J, \forall_{t} \in T \\
& Q_{S E_{k}}^{M i n} \leq Q_{S E_{k}}(t) \leq Q_{S E_{k}}^{M a x} ; \forall_{k} \in K, \forall_{t} \in T \\
& V_{n}^{M i n} \leq V_{n}(t) \leq V_{n}^{M a x} ; \forall_{n} \in N, \forall_{t} \in T \\
& -S_{\text {lnm }}^{M a x} \leq S_{\text {lnm }}(t) \leq S_{\text {lnm }}^{\operatorname{Max}} ; \forall l_{m n} \in L, \forall_{t} \in T
\end{aligned}
$$

Donde $\mathrm{K}, \mathrm{N}$ y L corresponden al conjunto de subestaciones, nodos y líneas, respectivamente. $\mathrm{P}_{\mathrm{SE}_{\mathrm{k}}}(\mathrm{t})$ corresponde a la potencia suministrada por la subestación $k$ en el intervalo de tiempo $t ; \rho_{\mathrm{SE}_{\mathrm{k}}}(\mathrm{t})$ Corresponde al costo de la energía suministrada a través de la subestación $\mathrm{k}$, en el intervalo de tiempo $\mathrm{t}$; $\mathrm{P}_{\mathrm{G}_{\mathrm{n}}}(\mathrm{t}), \mathrm{P}_{\mathrm{D}_{\mathrm{n}}}(\mathrm{t})$ y $\mathrm{P}_{\mathrm{n}}(\mathrm{t})$ corresponden a la potencia activa generadas, demandada y calculada en la barra $\mathrm{n}$, en el intervalo de tiempo $t$, respectivamente; $Q_{G_{n}}(t), Q_{D_{n}}(t)$ y $Q_{n}(t)$ corresponden a la potencia reactiva generadas, demandada y calculada en la barra $n$ en el intervalo de tiempo $t$, respectivamente. $V_{n}(t)$ corresponde al flujo de potencia aparente en la línea $l_{m n}$ en el intervalo de tiempo t. La ecuación (4) corresponde a la función objetivo del nivel inferior. Las ecuaciones (5) y (6) corresponden a las restricciones de balance de potencia activa y reactiva, respectivamente; las ecuaciones (7) y (8) corresponden a los límites de potencia activa suministrada por la subestación y las unidades de GD, respectivamente; la ecuación (9) corresponde al límite de potencia reactiva que se puede suministrar a la red a través de la subestación, finalmente, las restricciones (10) y (11) corresponden a los límites mínimos y máximos de tensión en barras y flujos de potencia en las líneas, respectivamente.

Las potencias activa y reactiva calculadas en cada barra están dadas por las ecuaciones (12) y (13).

$$
\begin{aligned}
& P_{n}=V_{n} \sum_{n \in N} V_{m}\left[g_{n, m} \cos \left(\theta_{n, m}\right)+b_{n, m} \operatorname{sen}\left(\theta_{n, m}\right)\right] \\
& Q_{n}=V_{n} \sum_{n \in N} V_{m}\left[g_{n, m} \operatorname{sen}\left(\theta_{n, m}\right)-b_{n, m} \cos \left(\theta_{n, m}\right)\right]
\end{aligned}
$$

Donde $\theta_{n, m}$ corresponde a la abertura angular entre las barras $n, m ; g_{n, m}$ y $b_{n, m}$ corresponden a las partes real e imaginaria del elemento $\mathrm{m}, \mathrm{n}$ de la matriz de admitancia nodal. El flujo de potencia aparente $S_{\operatorname{lmn}}$ está dado por los componentes de potencia activa y reactiva como se presenta en las ecuaciones (14) a (16).

$$
\begin{aligned}
& S_{l n m}=P_{l n m}+j Q_{l n m} \\
& P_{l n m}=V_{n}^{2} g_{n, m}-V_{n} V_{m} g_{n, m} \cos \left(\theta_{n, m}\right)-V_{n} V_{m} b_{n, m} \operatorname{sen}\left(\theta_{n, m}\right) \\
& Q_{l n m}=-V_{n}^{2} b_{n, m}+V_{n} V_{m} b_{n, m} \cos \left(\theta_{n, m}\right)-V_{n} V_{m} g_{n, m} \operatorname{sen}\left(\theta_{n, m}\right)
\end{aligned}
$$

El modelo descrito en (1)-(16) se diferencia de otros modelos y propuestas presentados en la literatura en que considera tres variables de optimización: ubicación, dimensionamiento y precio de contrato de la GD. La mayoría de los modelos que se encuentran disponibles en la literatura técncia como los presentados en (Yuanyuan et al., 2014), (Gozel y Hocaoglu, 2009 ) y (Elnashar et al., 2010) consideran la ubicación y el dimensionamiento de la GD, sin tener en cuenta el precio de contrato de la misma. Otros modelos, como los presetados en (Atwa y El-Saadany, 2011), (Khalesi et al., 2011) y (Hung et al., 2010) se enfocan en determinar únicamente la mejor ubicación de la GD.

Otro aspecto diferenciador del modelo propuesto consiste en que aborda el problema desde la perspectiva de programación binivel, considerando un mecanismo de acción-reacción entre dos agentes con diferentes intereses y un modelo $A C$ de la red. La mayoría de los artículos que se encuentran al respecto, abordan el problema de ubicar y/o dimensionar la GD exclusivamente desde el punto de vista de los beneficios al operador de red (Ochoa y Harrison, 2011), (Kumar y Gao, 2010); algunos autores, como se ilustra en (Ouyang et al., 2011) consideran el beneficio social. En (Soroudi et al., 2011) se consideran los beneficios, tanto para el operador de red, como para el propietario de la GD, pero desde un punto de vista de optimización multiobjetivo y no binivel. Finalmente, en (Rider et al., 2013) y (Lopez-Lezama et al., 2011) se considera un modelo de programación binivel pero no se utiliza un modelo AC de la red (se recurre a linealizaciones); además los trabajos mencionados anteriormente no optimizan los tres parámetros estudiados en el presente artículo. 


\section{METODOLOGÍA}

El modelo descrito anteriormente corresponde a un problema de programación no lineal entero mixto. Este problema es no convexo, multimodal y presenta el fenómeno de explosión combinatorial. Este tipo de problemas son típicamente abordados mediante técnicas de optimización metaheurística. En este caso se ha implementado un algoritmo genético especializado. Los Algoritmos Genéticos (AGs) son una familia de modelos computacionales inspirados en la evolución. En su implementación las propuestas de solución se modelan a través de una estructura de datos llamada cromosoma. Dada una población inicial, sobre esta son aplicados una serie de operadores con el objetivo de encontrar mejores alternativas de solución (Anaut et al., 2009). La principal ventaja de los AGs es la simplificación que estos permiten en la formulación y solución de problemas de optimización de alta complejidad.

\section{Codificación}

La codificación propuesta es ilustrada en la Fig. 2, en la cual cada individuo es representado por dos vectores. La longitud del vector indica el número de barras candidatas para ubicar GD. En este caso se tienen 9 barras candidatas. La selección del conjunto de barras candidatas se realiza a priori según criterios del planeador. Un criterio puede ser el precio marginal de las barras, el nivel de demanda, tensiones o cualquier otro criterio técnico. La primera fila del vector indica el tamaño de la GD y la segunda fila el precio de contrato. Según esta codificación, la Fig. 2 indica que se debe ubicar una unidad de GD de 1 MW en el nodo correspondiente a la casilla 2 , con un precio de contrato de $85 \$ / \mathrm{MWh}$; otra unidad de 3MW en el nodo correspondiente a la casilla 5 con un precio de contrato de $78 \$ / \mathrm{MWh}$ y así sucesivamente. La población inicial es generada de forma aleatoria, limitada por el número de barras de la red y el intervalo de discretización de los precios de oferta.

\begin{tabular}{|c|c|c|c|c|c|c|c|c|c|}
\hline 0 & 1 & 0 & 0 & 3 & 0 & 0 & 0 & 1 & 0 \\
\hline 0 & 85 & 0 & 0 & 78 & 0 & 0 & 0 & 80 & 0 \\
\hline
\end{tabular}

Fig. 2: Representación de la codificación propuesta para el AG.

\section{Evaluación de la función objetivo}

Para cada individuo de la población se debe calcular el valor de la función objetivo. En este caso la función objetivo corresponde a los lucros del propietario de la GD. Para cada individuo de la población se deben calcular los despachos óptimos, a un año, considerando la curva de duración de carga (demanda alta, media y baja con su respectiva duración). A partir de la energía que la GD vendería al operado de red se calculan sus lucros usando la ecuación (1). Para la solución de los despachos óptimos se utiliza el software Matpower (Zimmerman et al., 2011).

\section{Selección}

La selección es el operador que permite escoger los individuos de la población actual que participan en la generación de un nuevo individuo. El proceso de selección implementado usa una competencia basada en dos torneos. En cada tornero, $\mathrm{k}$ individuos son seleccionados aleatoriamente de la población actual, y aquel con mejor función objetivo es seleccionado para generar nuevos descendientes. Los dos individuos seleccionados deben ser diferentes. Posteriormente, ambos individuos deben pasar a la fase de recombinación.

\section{Recombinación}

Los individuos escogidos en el proceso de selección son sometidos al operador de recombinación. La recombinación consiste en cambiar parcelas de las configuraciones escogidas en el proceso de selección para formar nuevas configuraciones. En el algoritmo propuesto se utiliza una recombinación de punto simple.

\section{Mutación}

El individuo seleccionado en la recombinación debe pasar por la etapa de mutación. En esta etapa una posición del cromosoma es aleatoriamente seleccionada con una probabilidad específica, y su información es cambiada. La mutación es hecha en el tamaño de la unidad o en el precio de contrato con la misma probabilidad. En ambos casos, la información correspondiente es cambiada de forma aleatoria dentro de un intervalo predeterminado. 


\section{Búsqueda local}

En el AG implementado, en cada iteración se realiza una búsqueda local para mejorar la solución actual. La fase de búsqueda local es efectuada en la primera o segunda línea del cromosoma con igual probabilidad. Si se hace en la primera línea, entonces se modifica el tamaño de la GD y se evalúan los nuevos individuos, buscando mejores respuestas. Si se hace en la segunda línea, esa misma búsqueda es hecha cambiando el precio de contrato de las unidades de GD dentro de un intervalo especificado. Si un individuo mejor es encontrado, entonces este individuo es seleccionado para pasar para la etapa siguiente. La fase de búsqueda local permite una evolución más eficiente del AG, y con la mutación, ayuda al algoritmo a escapar de óptimos locales.

\section{Substitución de la población}

Al contrario de los AGs tradicionales, el AG especializado propuesto en este artículo substituye en cada interacción, solamente un individuo. En este caso, todos los individuos representados por la codificación propuesta son factibles. Así, el individuo candidato solamente puede substituir un individuo de la población actual si fuese mejor y diferente de este. Esta lógica de substitución preserva tanto los mejores individuos como también la diversificación de la población y evita la convergencia prematura del algoritmo. El proceso es interrumpido si la incumbente (la mejor solución encontrada) no cambia después de un número predefinido de generaciones, o cuando el número máximo de generaciones es alcanzado. En la Fig. 3 se ilustra el flujograma del AG propuesto.

\section{PRUEBAS Y RESULTADOS}

Para probar la efectividad y robustez del AG propuesto se utilizó el sistema de 85 barras ilustrado en la Fig. 4. Para todos los cálculos se ha supuesto un costo de operación de la GD de 60 \$/MWh y costos anualizados de inversión de $\$ 50000$ por MW instalado. En cuanto al dimensionamiento se consideraron unidades de 0.5, 1.0, 1.5 y 2.0 MW. Los datos de línea del sistema se pueden consultar en (Buitrago, 2015). La distribución de carga de este sistema se ilustra en la Fig. 5 y la duración de carga en la Fig. 6 . En cada uno de los niveles de carga se indica el precio de la energía en el mercado mayorista en \$/MWh.

Las barras candidatas para ubicar GD se seleccionaron en función de sus precios marginales, calculados a partir de un flujo de potencia sin GD. Dada la característica radial del sistema se pudo identificar que los precios marginales son mayores en las últimas barras del sistema. Por lo tanto se seleccionaron 25 nodos candidatos de las ramas más alejadas de la subestación. Las barras candidatas son: [ 12, 14, 15, 41, 42, $43,46,47,51,53,54,55,56,61,62,63,65,67,73,74,77,81,82,84,83]$.

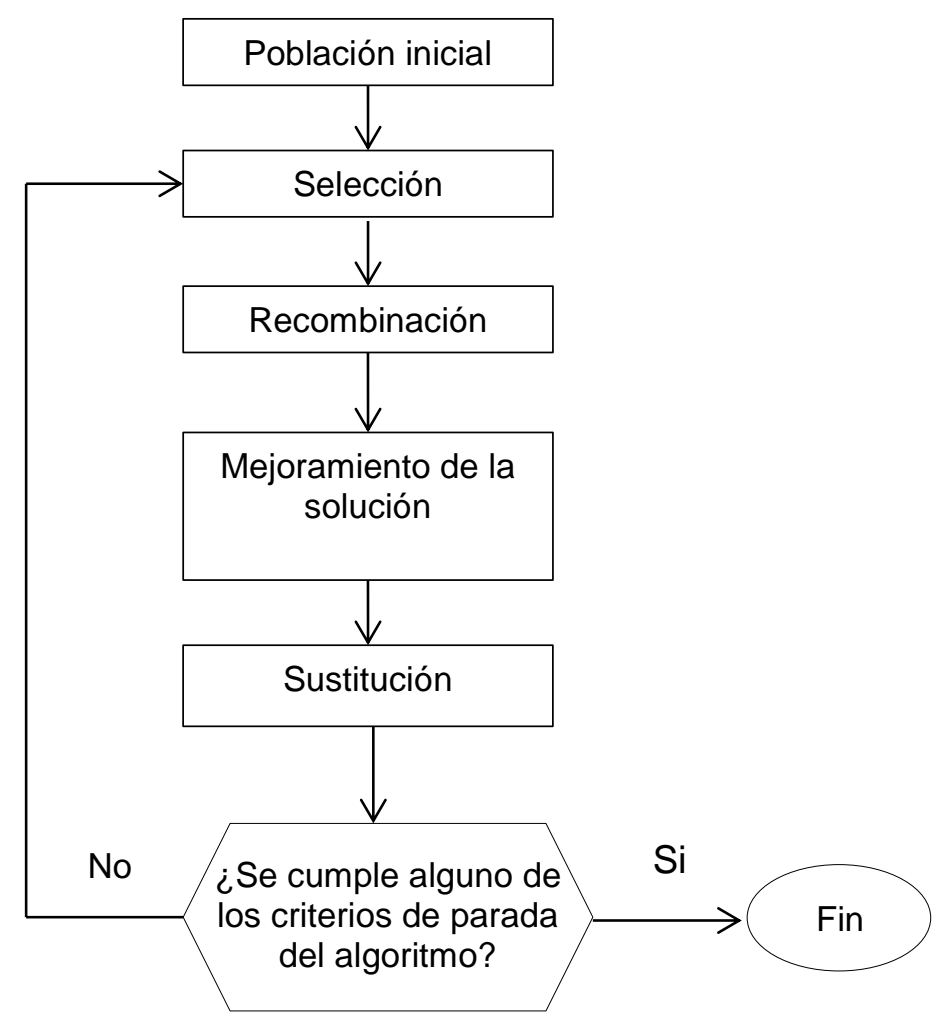

Fig. 3: Flujograma del AG propuesto. 


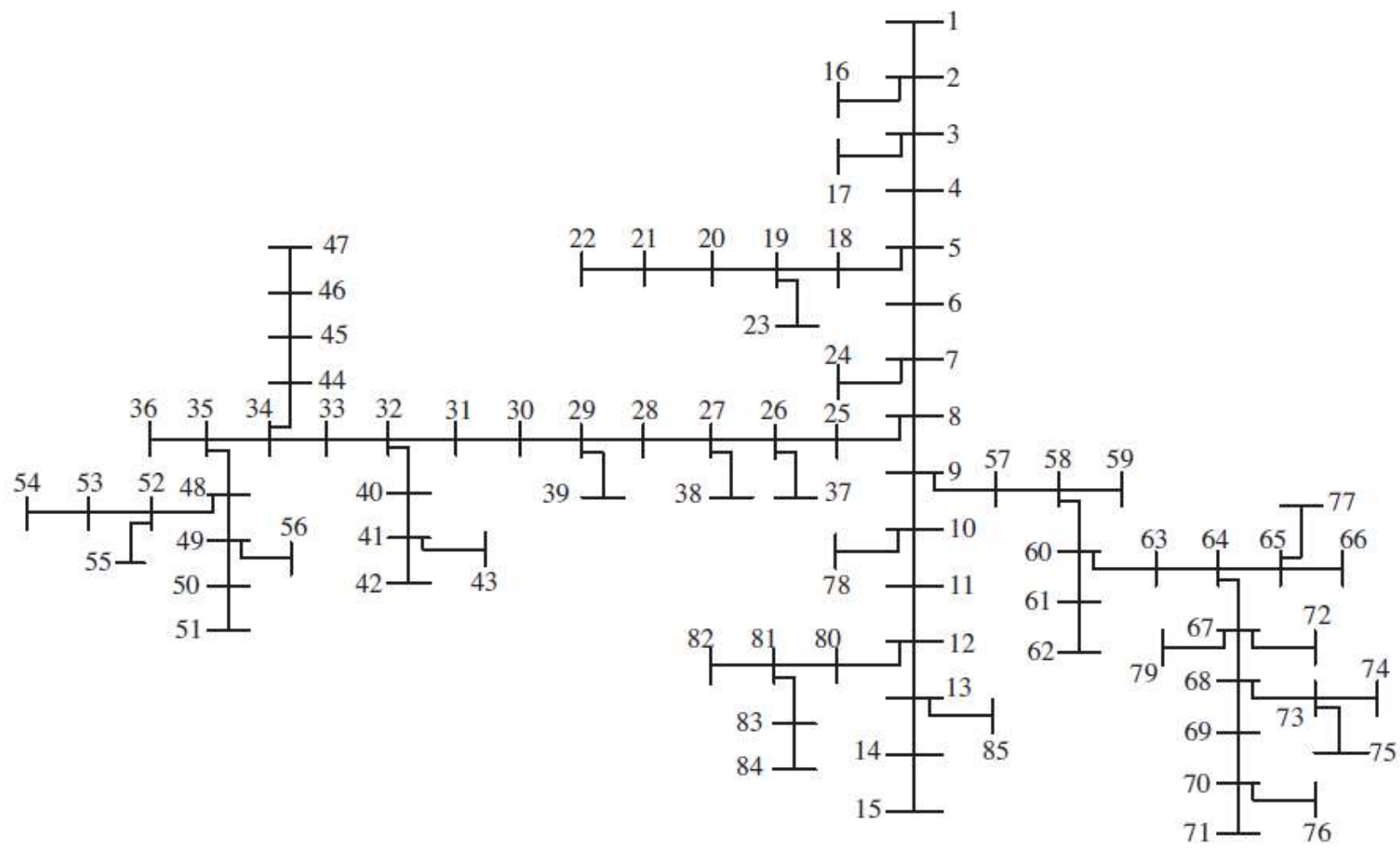

Fig. 4: Sistema de prueba de 85 barras

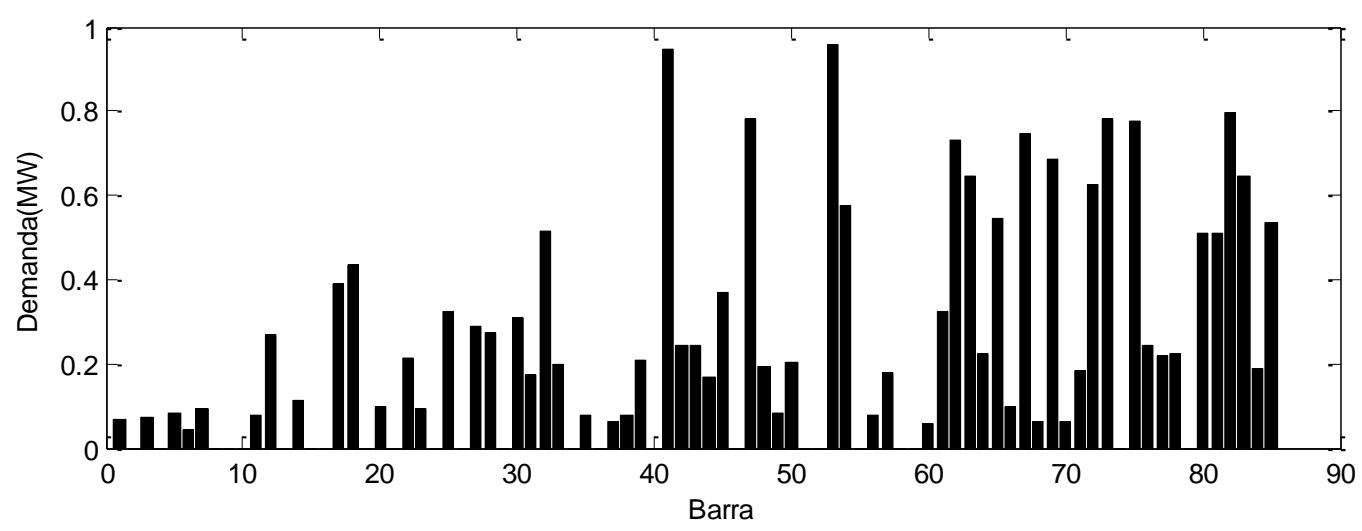

Fig. 5: Distribución de demanda sistema de 85 barras

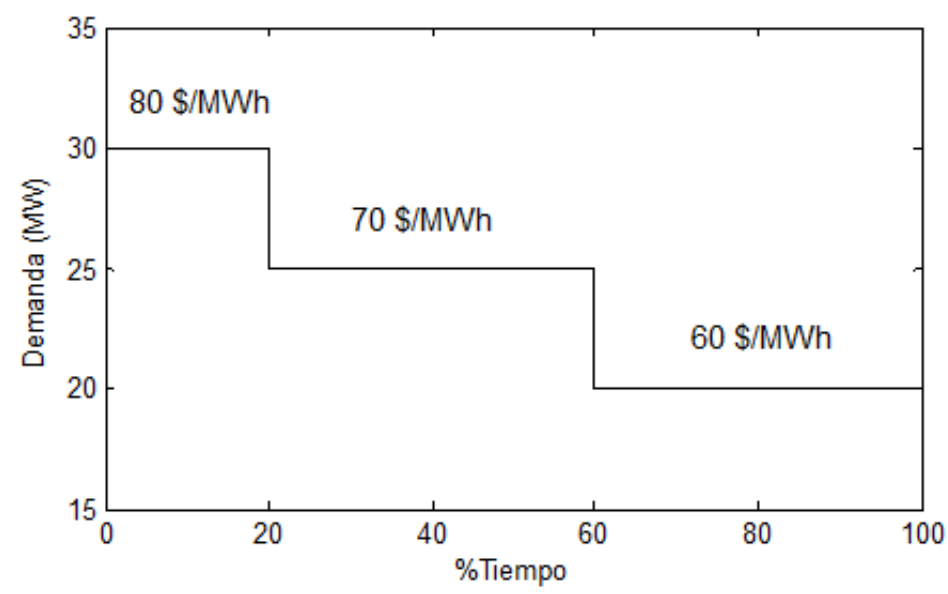

Fig. 6: Curva de duración de carga para el sistema de 85 barras 
Después de rodar múltiples casos y de ajustar parámetros se encontraron mejores soluciones con poblaciones iniciales grandes (100 individuos). En este caso el algoritmo tomó entre 80 y 100 iteraciones para converger. En el AG se ajustó la probabilidad de hacer búsqueda local en precios en 0.5 , tasa de mutación de 0.2 y una tasa de recombinación de 10 individuos. Para la búsqueda local en precios, se exploraron soluciones vecinas que diferían del precio actual en más o menos $6 \$ / \mathrm{MWh}$. Para la búsqueda local en tamaño se exploraban soluciones vecinas variando todos los posibles tipos de tamaño de GD, desde 0 hasta $2 \mathrm{MW}$ en intervalos de $0.5 \mathrm{MW}$.

Los mejores resultados obtenidos con el AG se resumen en la Tabla 1. Se puede observar que las soluciones son de calidad similar y contemplan la ubicación de 4 o 5 unidades de GD con capacidades que oscilan entre 0.5 y $2 \mathrm{MW}$. Se encontró que la máxima participación de la GD es de $9.5 \mathrm{MW}$ (un $31.6 \%$ de la demanda máxima). Soluciones con más capacidad de GD instalada no resultaron ser de buena calidad debido al costo de inversión. En la Fig. 7 se ilustra el desempeño del AG para los casos reportados en la Tabla 1.

Las soluciones encontradas representan un equilibrio entre los intereses de ambos agentes. El beneficio del operador de red se ve reflejado principalmente en los ahorros en la compra de energía (ver Tabla 1) y de forma secundaria, en la reducción de pérdidas y mejoramiento del perfil de tensiones. En la Fig. 8 se ilustran las pérdidas de potencia para los tres niveles de carga, con y sin GD. Se puede observar que la GD contribuye significativamente a la reducción de pérdidas en los niveles de carga Medio y Alto. En el nivel de carga Bajo la GD no es despachada por el operador de red y por lo tanto, no contribuye a la reducción de pérdidas. En la Fig. 9 se ilustra el perfil de tensiones de la red para los resultados de la Prueba 1 en el nivel de carga alto. Se puede apreciar que la introducción de GD tiene un impacto positivo en las tensiones de la red.

Tabla 1: Mejores resultados obtenidos con el AG

\begin{tabular}{|c|c|c|c|c|}
\hline \multicolumn{2}{|c|}{ Mejores resultados } & Prueba 1 & Prueba 2 & Prueba 3 \\
\hline \multirow{3}{*}{ GD1 } & Ubicación (barra) & 54 & 47 & 12 \\
\hline & Precio (\$/MWh) & 78 & 80 & 80 \\
\hline & Tamaño (MW) & 1.5 & 2 & 1.5 \\
\hline \multirow{3}{*}{ GD2 } & Ubicación (barra) & 56 & 54 & 54 \\
\hline & Precio (\$/MWh) & 78 & 80 & 80 \\
\hline & Tamaño (MW) & 1.5 & 2 & 1.5 \\
\hline \multirow{3}{*}{ GD3 } & Ubicación (barra) & 63 & 81 & 56 \\
\hline & Precio (\$/MWh) & 79 & 81 & 79.5 \\
\hline & Tamaño (MW) & 2 & 2 & 2 \\
\hline \multirow{3}{*}{ GD4 } & Ubicación (barra) & 73 & 63 & 81 \\
\hline & Precio (\$/MWh) & 80 & 81 & 78 \\
\hline & Tamaño (MW) & 2 & 2 & 2 \\
\hline \multirow{3}{*}{ GD5 } & Ubicación (barra) & $N / A$ & 65 & $N / A$ \\
\hline & Precio (\$/MWh) & $\mathrm{N} / \mathrm{A}$ & 81 & $\mathrm{~N} / \mathrm{A}$ \\
\hline & Tamaño (MW) & $N / A$ & 1.5 & $\mathrm{~N} / \mathrm{A}$ \\
\hline \multicolumn{2}{|c|}{ Lucro del propietario de GD (\$) } & 351676 & 353428 & 354009 \\
\hline \multicolumn{2}{|c|}{ Ahorro del operador de Red $(\$)$} & 433753 & 412729 & 417109 \\
\hline
\end{tabular}




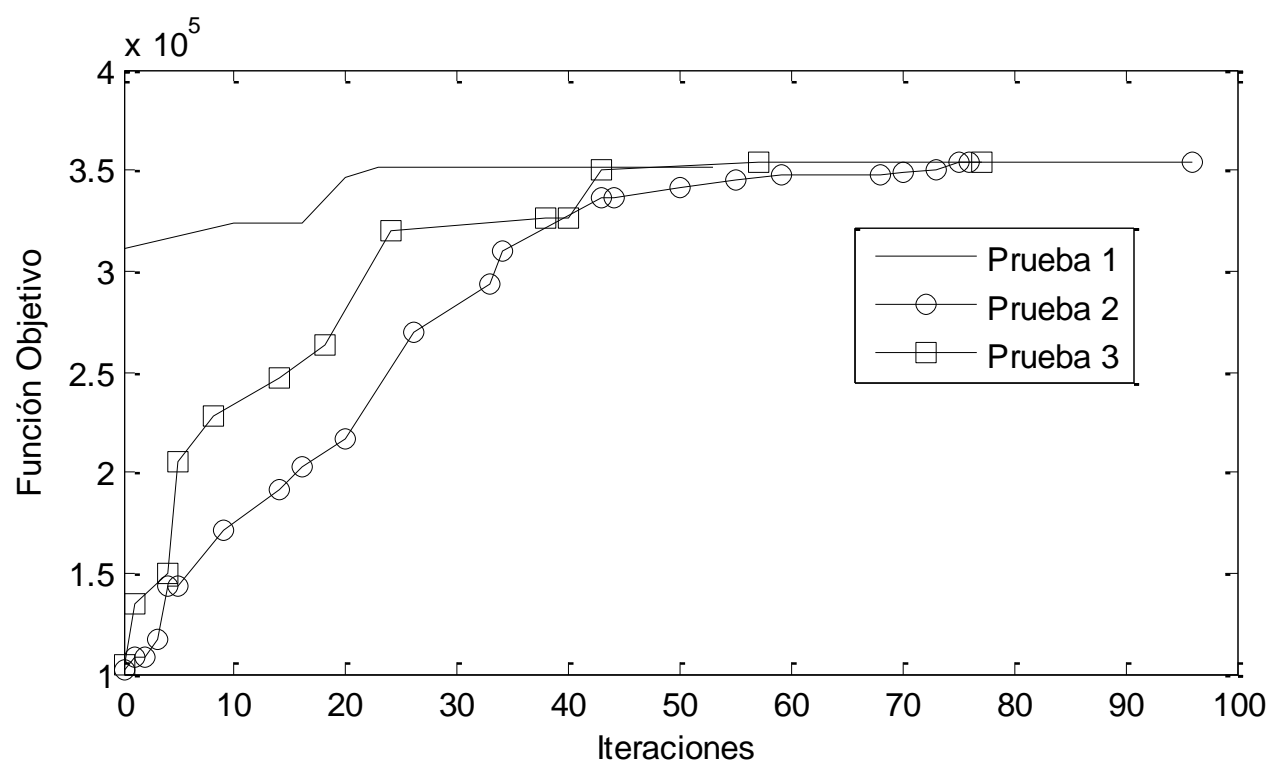

Fig. 7: Desempeño del AG para diferentes pruebas

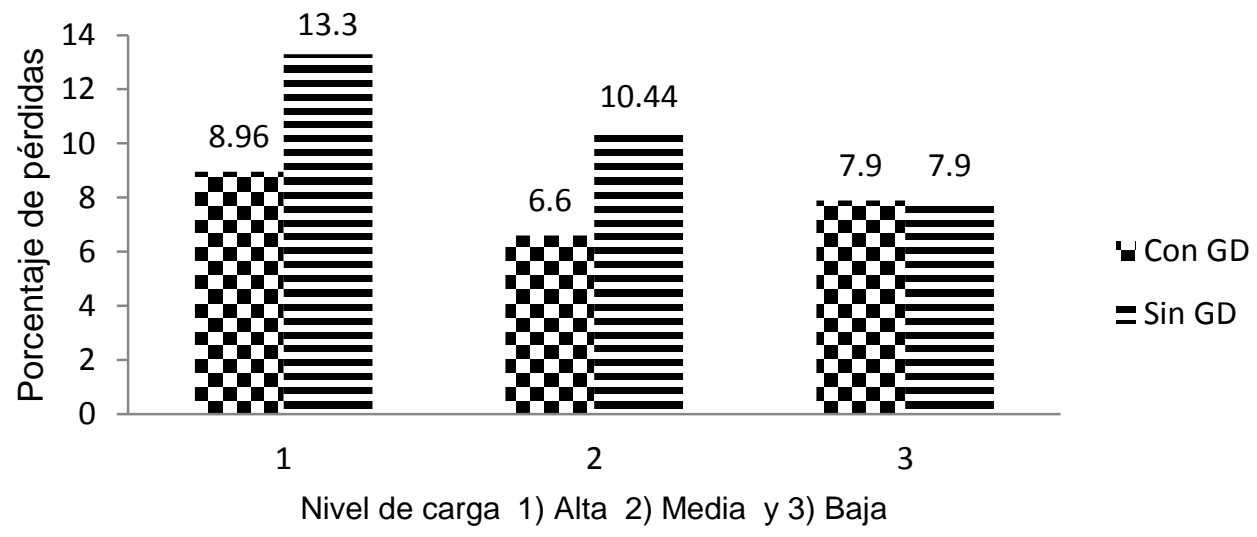

Fig. 8: Pérdidas del sistema con y sin GD

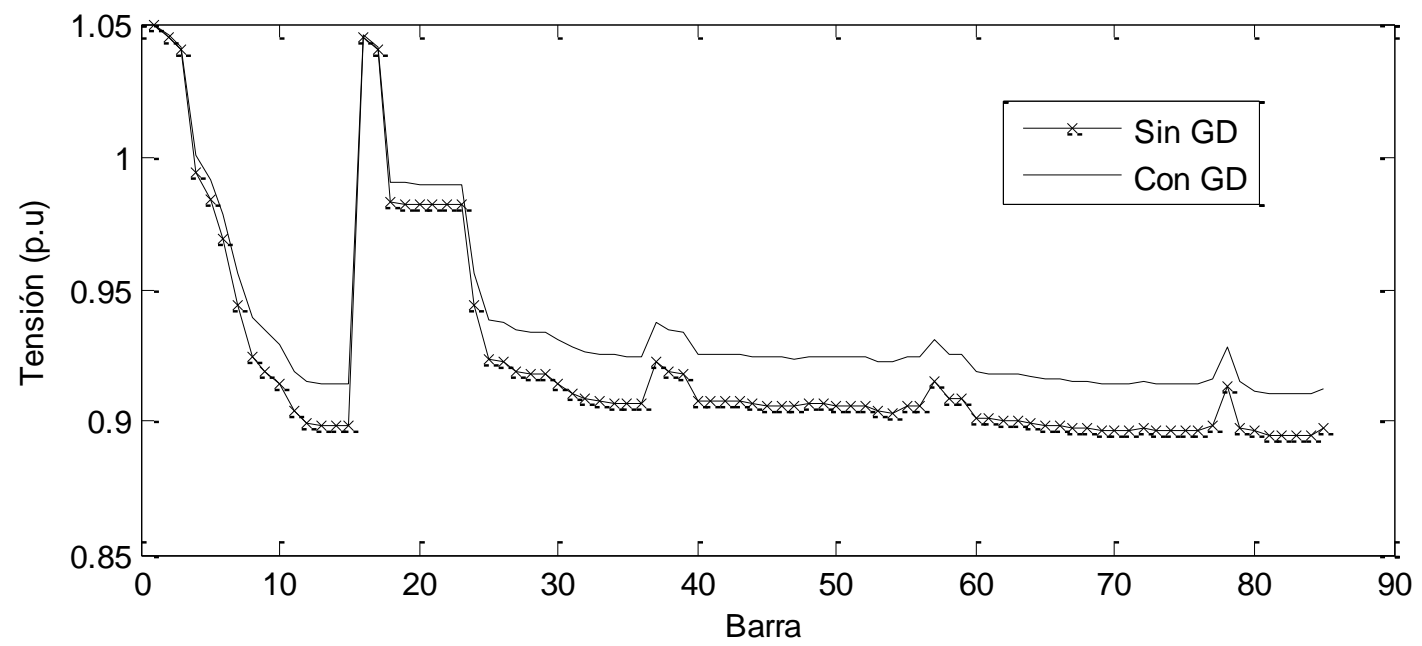

Fig. 9: Perfil de tensiones con y sin GD (Prueba 1 nivel de carga Alto) 


\section{CONCLUSIONES}

Se desarrolló un modelo de programación binivel para determinar la ubicación, precio de contrato y dimensionamiento óptimo de varias unidades de GD pertenecientes a un único propietario. El modelo propuesto combina en un único problema de optimización, la maximización de los lucros que procura el propietario de la GD y la minimización de los pagos buscada por el operador de la red. Desde el punto de vista de la reglamentación de los mercados de energía, el modelo propuesto suministra incentivos económicos adecuados, tanto para el propietario de la GD, como para operador de red. Esto sucede debido al hecho de que los intereses de ambos agentes (el operador de la red y el propietario de la GD) son considerados de forma simultánea en el proceso de optimización. Una de las ventajas del modelo propuesto es que permite al propietario de la GD adelantarse a la reacción del operador de red. Esto es hecho considerando el problema de optimización del operador de red como un conjunto de restricciones del problema de optimización del propietario de la GD.

El modelo de programación binivel propuesto considera variables enteras, es no convexo y multimodal (presenta múltiples soluciones subóptimas). Por lo tanto fue solucionado usando una metaheurística. La técnica utilizada es un algoritmo genético. La principal ventaja del método propuesto consiste en la posibilidad de incluir variables enteras en el modelo binivel. El problema del nivel inferior es solucionado de forma explícita en cada iteración mediante un despacho óptimo.

Dada la naturaleza del problema solo se consideran tecnologías de GD que son despachables o controlables. Esto se da porque es el operador de la red de distribución es quién decide cuanta energía necesita de la GD y en qué momento. El propietario de la GD está sujeto a esta condición y por lo tanto debe tener disponibilidad de energía para suplir las necesidades del operador de red.

\section{AGRADECIMIENTOS}

Los autores agradecen a la Universidad de Antioquia (UdeA) por el apoyo del proyecto "Sostenibilidad 20142015" para el desarrollo de este trabajo.

\section{REFERENCIAS}

Abu-Mouti, F.S., y El-Hawary. M.E., Optimal distributed generation allocation and sizing in distribution systems via Artificial Bee Colony Algorithm, IEEE Transactions on Power Delivery, 26(4): 2090-2101, (2011)

Aghaei, J., Akbari M.A., Roosta, A., Gitizadeh, M., y Niknam, T., Integrated renewable-conventional generation expansion planning using multiobjective framework. IET Generation Transmission and Distribution: 6(8), 773-784, (2012)

Agudelo, L., López-Lezama, J.M., y Muñoz, N., Análisis de vulnerabilidad de sistemas de potencia mediante programación binivel. Revista Inf. Tecnol. Cit: 25(3), 103-114, (2009)

Anaut, D., Di Mauro, G., Meschino, G., y Suarez, J., Optimización de Redes Eléctricas Mediante la Aplicación de Algoritmos Genéticos, Revista Inf. Tecnol. Cit: 20(4), 137-148, (2009)

Atwa, Y.M. y El-Saadany, E.F., Probabilistic approach for optimal allocation of wind-based distributed generation in distribution systems, IET Renewable Power Generation., 5(2), 79-88, (2011)

Buitrago, L.F., Ubicación, dimensionamiento y precio de contrato óptimo de GD, Tesis de Magister, Facultad de Ingeniería, Universidad de Antioquia, Colombia, (2015)

Carrión, M., Arroyo, J.M., y Conejo A.J., A bilevel stochastic programming approach for retailer futures market trading. IEEE Transactions on Power Systems: 24(3), 1446-1456, (2009)

Elnashar, M.M., El Shatshat, R., and Salama, M.M. Optimum siting and sizing of a large distributed generator in a mesh connected system, Electric Power Systems Research., 80(6), 690-697, (2010)

Garcés, L., Conejo A.J., Garcia-Bertrand, R., y Romero, R. A bilevel approach to transmission network expansion planning wihin a market environment. IEEE Transactions on Power Systems: 24(3), 1513-1522, (2009)

Georgilakis, P.S. y Hatziargyriou, N.D., Optimal distributed generation placement in power distribution networks: models, methods and future research, IEEE Transactions on Power Systems, 28(3), 3420-3428, (2013) 
Gozel, T., y Hocaoglu M.H., An analytical method for the sizing and sitting of distributed generators in radial systems, Electric Power Systems research, 79(6), 912-918, (2009)

Hung, D.Q., Mithualananthan, N., y Bansal R.C., Analytical expressions for DG allocation in primary distribution networks, IEEE Transactions on Power Systems, 25(3), 814-820, (2010)

Khalesi, N., Rezaei, N. y Haghifam, M.R. DG allocation with application of dynamic programming for loss reduction and reliability improvement, International Journal of Electrical Power \& Energy Systems., 33(2), 288-295, (2011)

Kumar, A. y Gao, W., Optimal distributed generation location using mixed integer non-linear programming in hybrid electricity markets. IET Generation Transmission and Distribution: 4(2), 281-298, (2010)

Lopez-Lezama J.M., Padilha-Feltrin A., Contreras A. y Muñoz J.I., Optimal contract pricing of distributed generation in distribution systems, IEEE Transactions on Power Systems, 26(1), 128-136, (2011)

Mobarakeh, A.S., Rajabi-Ghahnavieh, A., y Zahedian, A., A game theoretic framework for DG optimal contract pricing. Proceedings of the $14^{\text {th }}$ IEEE/PES Innovative Smart Grid Technologies Conference, 1-5, Lyngby, Denmark, 6-9, October (2013)

Ochoa, L.F. y Harrison, G.P. Minimizing Energy Losses: Optimal Accommodation and Smart Operation of Renewable Distributed Generation, IEEE Transactions on Power Systems, 26(1), 198-205, (2011)

Ouyang, W., Cheng, H., Zhang, X. and Li, F. Evaluation of distributed generation connecting to distribution network based on long-run incremental cost, IET Generation, Transmission \& Distribution., 5(1), 561-568, (2011)

Rider, M., Lopez-Lezama, J.M., Contreras, J., Padilha, A., Bilevel Programming Approach for Optimal Location and Contract Pricing of Distributed Generation in Radial Distribution Networks, IET Generation Transmission and Distribution., 7(7), 724-734, (2013)

Shaaban, M.F, Atwa, Y.M., y El-Saadany, E.F., GD allocation for benefit maximization in distribution networks. IEEE Transactions on Power Systems: 28(2), 639-649, (2013)

Singh, D., y Verma K.S., Multiobjective optimization for GD planning with load models. IEEE Transactions on Power Systems: 24(1), 427-436, (2009)

Soroudi, A., Ehsan, M., Caire, R., y Hadjsaid, N., Hybrid immune-genetic algorithm method for benefit maximization of distribution network operators and distributed generation owners in a deregulated environment., IET Generation Transmission \& Distribution., 5(9), 961-972, (2011)

Yuanyuan, Z., Yiran, A. y Qian, A., Research on size and location of distributed generation with vulnerable node identification in the active distribution network. IET Generation Transmission and Distribution: 8(11), 1801-1809, (2014)

Zimmerman, R.D., Murillo-Sánchez, C.E., and Thomas R.J., Matpower: a steady-state operations planning and analysis tools for power systems research and education., IEEE Transactions on Power Systems., 26(1),12-19, (2011) 
